\title{
Time of Day Effect on Soccer-Specific Field Tests in Tunisian Boy Players
}

\author{
Adnene Gharbi ${ }^{1,2}$, Liwa Masmoudi ${ }^{1}$, Sleheddine Ghorbel ${ }^{1}$, Noureddine Ben Saïd ${ }^{3}$, \\ Riadh Maalej ${ }^{1}$, Zouhair Tabka ${ }^{2}$, Monia Zaouali ${ }^{2}$ \\ ${ }^{1}$ High Institute of Sport and Physical Education, Sfax University, Sfax, Tunisia \\ ${ }^{2}$ Laboratory of Cardio-Circulatory, Respiratory, Metabolic and Hormonal Adaptations to \\ the Muscular Exercise, Faculty of Medicine Ibn El Jazzar, Sousse, Tunisia \\ ${ }^{3}$ College of Sport Sciences \& Physical Activity, King Saud University, Riyadh, Saudi Arabia \\ Email: adnenegharbi@yahoo.fr
}

Received March $7^{\text {th }}, 2013$; revised April 10 $0^{\text {th }}, 2013$; accepted April 25 $5^{\text {th }}, 2013$

Copyright (C) 2013 Adnene Gharbi et al. This is an open access article distributed under the Creative Commons Attribution License, which permits unrestricted use, distribution, and reproduction in any medium, provided the original work is properly cited.

\begin{abstract}
The purpose of this study was to investigate the diurnal variation in some specific skills performance during field testing in boy's footballer. In a balanced and randomized study design, 15 boys (mean $\pm \mathrm{SD}$; age $12.7 \pm 1.1$ years; height $1.54 \pm 9.1 \mathrm{~m}$; body mass $45.1 \pm 3.2 \mathrm{~kg}$ ) participated in the study. Subjects performed some specific soccer skills (kicking accuracy, ball control with the body, ball control with the head, coordination test, zigzag running (i.e., agility), zigzag with the Ball (i.e., dribbling) and the skill index (i.e., the ratio of the zigzag running without and with the ball) at two different times of day: 07:00 and 17:00 h. Moreover, intra-aural temperature was measured at both time-of-days. Intra-aural temperature was higher in the evening than the morning $(P<0.01)$. Likewise, a significant time-of-day effect was found for dribbling $(P<0.05)$ and agility $(P<0.001)$ with higher performances observed at 17:00 h. However, no significant differences were noticed between 07:00 and 17:00 h for the skill index, kicking accuracy, ball control with the body, ball control with the head and coordination tests. The intra-aural temperature was found to be significantly correlated with the speed of dribbling and agility $(P<0.001)$. In conclusion, a diurnal variation in intra-aural temperature, agility and dribbling were shown to be present. No time-of-day effect was observed for the coordination, skill index, kicking accuracy and juggling performance.
\end{abstract}

Keywords: Circadian Rhythm; Intra-Aural Temperature; Boy Players; Field Tests; Soccer Skills

\section{Introduction}

Football games may take place at various times throughout the day whilst training may be held in the morning or afternoon. Despite its popularity as a sport, few scientific studies have focused on the circadian variation in soccer players' performance. For instance, some investigators, who are interested in soccer performance, reported that aerobic (Chtourou et al., 2012b), anaerobic performances (Chtourou et al., 2012a, 2012b; Hamouda et al., 2012) and repeated sprint ability (Chtourou et al., 2012b) fluctuate with time of day. Indeed, peak and mean power during maximal cycling test (Chtourou et al., 2012b; Hamouda et al., 2012; Aloui et al., 2012), maximal voluntary contraction (Aloui et al., 2012), Flexibility (Reilly et al., 2007), hand-grip strength (Reilly et al., 2007), total work during the repeated sprint ability test (Chtourou et al., 2012b), total distance and maximal aerobic velocity during the Yo-Yo test (Chtourou et al., 2012b) were significantly higher in the evening than in the morning. Most of studies showed that peak soccer performances have been found to occur in the early evening corresponding to the peak of the body temperature rhythm. Furthermore, worst performance has been found in the morning (Aloui et al., 2012; Reilly et al., 2007; Bernard et al., 1998;
Melhim et al., 1993). Circadian rhythm in exercise performance for soccer players with morning lows and evening peaks are common findings in many laboratory tests performed on cycle ergometer (Chtourou et al., 2010a, 2012b; Hamouda et al., 2012; Aloui et al., 2012). However, each test is measured in athletes, it is important that the test include the activity pattern of the specific sport. Therefore, testing soccer should be performed on field and including some soccer skills, as opposed to a cycle ergometer to improve the specificity in the activity patterns in the football. Sport science support workers can, therefore, use field tests to evaluate specific aspects of soccer performance, which may provide a better indication of the ability to perform in a soccer match than laboratory-based evaluations. There are a wide range of skills which form the foundation of soccer performance. Other skills are important in the game but have received much less attention. The actual motor tasks of passing, controlling, dribbling, starting, stopping, changing direction and shooting the ball are all important skills in soccer but have received little detailed analysis. To date, a little attention has been paid to circadian and diurnal rhythms in skill measures, particularly those specifically related to a particular soccer game.

Previous research indicated that many aspects of soccer per- 
formance vary according to a circadian rhythm (Reilly et al., 2004a, 2004b, 2007). Football-specific skills of juggling performance showed significant diurnal variation (peak at 16:00 h), whereas performance on the wall-volley test tended to peak at 20:00 h (Reilly et al., 2004a, 2007). Nevertheless, no time-ofday effect was described in the dribbling test (Reilly et al., 2004a, 2007). Diurnal variation was also described in some football-specific tests, including dribbling time and chip test performance, being more accurate in the evening (Reilly et al., 2004b). Reilly et al. (2007) indicated that adult football players perform at an optimum between 16:00 and 20:00 h not only for football-specific skills but also for some physical performances. Reilly et al. (2004a, 2004b, 2007) investigated the diurnal variation of skill measures only in adult subjects. In this context, various chronobiological studies suggest that the temporal order of children differs from that of adults (Huguet et al., 1995).

To our knowledge, there is no study available in soccer has been paid to diurnal variation in skill measures, particularly those specifically related to a particular game in boys. Consequently, the aim of the present study was to assess the effects of time-of-day in some specific skills performance during field testing in boy's footballer.

\section{Protocol Experimental}

Subjects

15 boys belonging to an academy of soccer (Maracana) and having $4.2 \pm 1.1$ years of playing experience were recruited to participate in our study. Subjects and their parents were informed about the experimental procedure. The study was conducted according to the declaration of Helsinki and the protocol was approved by the local Ethics Committee. Moreover, all the subjects were classified as "neither type" from their responses to the self-assessment questionnaire of Horne and Ostberg (1976), which determines morningness-eveningness. The mean age, height and body mass of the subjects were $12.7 \pm 1.1$ years, $1.54 \pm 9.1 \mathrm{~m}$ and $45.1 \pm 3.2 \mathrm{~kg}$, respectively.

\section{Experimental Design}

Participants arrived $1 \mathrm{~h}$ before the start of the test and lay down and relaxed. Then the subject's intra-aural temperature was measured by an infrared ear thermometer (ThermoScan IRT 4520, Braun GmbH, Kronberg, Germany). After a standardized warm-up of 10 - 15 min, subjects performed some specific soccer skills in this order: kicking accuracy, ball control with the body, ball control with the head, coordination tests, Zigzag Test (i.e., agility), Zigzag with the Ball (i.e., dribbling) and the skill index (i.e., the ratio of the zigzag running without and with the ball). Subjects performed two trials of each test (except accuracy test), with a 3 minute interval, and the best performances in each test were used for analysis. There was a 5 minute rest session between the two tests. The measurements were taken at two different times of the day: 07:00 and 17:00 hr in a randomized order over two days with a minimum recovery period of $36 \mathrm{hr}$. Tests were conducted outdoors on a playing field of artificial grass where the mean ambient temperature and relative humidity were $24.5^{\circ} \mathrm{C} \pm 0.2^{\circ} \mathrm{C}, 53.1 \% \pm 0.2 \%$ and $23.9^{\circ} \mathrm{C} \pm 0.2^{\circ} \mathrm{C}, 52.7 \% \pm 1 \%$ for the morning and the evening, respectively. These conditions indicated that the weather was slightly modified throughout the study. The subjects were asked to avoid all vigorous activity before each test, to sleep normally, to wear the same sportswear and shoes for all tests. All the subjects had been familiarized with the tests before participating in the study.

\section{Tests Description}

- Kicking accuracy: every subject used dominant foot to perform 10 kicks. We constructed a plywood target measuring system with $243.5 \mathrm{~cm}$ wide $\times 122 \mathrm{~cm}$ high. Carbon paper was applied to the surface of the target. The distance between target and ball position is $610 \mathrm{~cm}$. The distance between bull's-eye and ground is $120 \mathrm{~cm}$ (Finnoff et al., 2002). To determine the accuracy of the kick, we analyzed video recordings of each trial. The distance between ball mark and bulls-eye after each kick was measured. The mean error of the 10 kicks was retained for analysis, so that the smaller the score, the better the KA (Finnoff et al., 2002).

- Ball control with the body: Within a $9 \times 9 \mathrm{~m}$ square, the player had to keep the ball in the air without using the arms or hands. The score recorded was the number of the ball before it fell to the floor. Counting stopped when the ball hit the floor, the participant moved out of the square or he touched the ball with the arms or hands. One trial was administered, although the participant could start the trial again if he failed to contact the ball three times in the initial attempt (Malina et al., 2005).

- Ball control with the head: Within a $9 \times 9 \mathrm{~m}$ square, the player had to keep the ball in the air using only the head. The score recorded was the number of hits of the ball before it fell to the floor. Counting stopped when the ball hit the floor, the participant moved out of the square or he touched the ball with any part of the body except the head. One trial was administered, although the participant was allowed to start the trial again if he failed to contact the ball three times in the initial attempt (Malina et al., 2005).

- Coordination test: This test enables to evaluate the football coordination of various parts of the body. The researcher throws the ball to the player from a distance of $5 \mathrm{~m}$. The player starts to play with ball in the order of chest-foot-head, head-left foot-right foot and foot-chest-head. The researcher measures them and every successful play brings 1 point (Rosch et al., 2000).

- Zigzag Test. This test assessed running agility from changes in direction. A zigzag course consisted of $5 \mathrm{~m}$ sections set out at 100 angles. The selection of this test was based on rapid acceleration, deceleration, and balance control required for short running time, which represented the result of the test (Little et al., 2005).

- Zigzag with the Ball. The ability to control the ball while changing direction was assessed. Subjects were instructed to run with the ball as fast as possible along the same zigzag path used in the previous test (Mirkov et al., 2008). The time in Zigzag Test and Zigzag with the Ball (all running tests) measurement is made with a stopwatch in units of 0.1 seconds (Rosch et al., 2000).

- Skill Index. The ratio of the results obtained from the zigzag test without and with the ball was calculated. A higher index (i.e., a smaller relative increase in the zigzag running time when the ball had to be controlled) was interpreted as a higher skill of controlling the ball (Mirkov et al., 2008). 


\section{Statistical Analysis}

All results are expressed as mean $( \pm \mathrm{SD})$. After checking the normality of the distribution with the Komolgorov-Smirnov test, we used a paired Student's t-test to investigate differences between morning and evening for all measurement. Pearson correlations were used to assess the relationships between variables. Statistical significance was set at $P<0.05$.

\section{Results}

A significant diurnal variation was observed for temperature when measured at 7:00 $\mathrm{h}$ and 17:00 h. $(P<0.01)$. The intra-aural temperature was $0.68^{\circ} \mathrm{C}$ higher in the evening (Figure 1) than the morning. Results showed a significant main effect of time of day for Zigzag Test (i.e., agility) $(P<0.001)$, and Zigzag with the Ball (i.e., dribbling) $(P<0.05)$ : the performances were better in the evening than in the morning. The diurnal gains were $6.7 \% \pm 5.5 \%$ and $4.6 \% \pm 5.9 \%$ for Zigzag Test and Zigzag with the Ball respectively. However, there was no significant time of day effect for other skills including juggling performance (Ball control with the body and with the head), coordination tests, skill index and kicking accuracy (Table 1).

Interestingly, as reported in Figure 2, the speed for agility and dribbling were positively and significantly correlated with intra-aural temperature $(\mathrm{r}=0.67$ and $\mathrm{r}=0.76$, respectively, $P<$ 0.001).

\section{Discussion}

The aim of the current study was to investigate diurnal variation in some specific skills performance in boy's footballers. The main finding was the increase of agility and dribbling in the evening. The measures showed that the best performance was at 17:00 $\mathrm{h}$ and the intra-aural temperature fluctuates concomitantly. However, the diurnal variation in the juggling performance (Ball control with the body and ball control with the head) just failed to reach significance. The coordination tests, skill index and kicking accuracy did not show a significant time-of-day effect.

Our results showed a significant diurnal variation in intra-aural temperature as it was described in previous studies (Reilly et al., 2004a, 2004b, 2007) (Figure 1). Body temperature as a marker of circadian rhythms, shows a diurnal variation with higher values recorded at 17:00 h (Figure 1). Moreover,

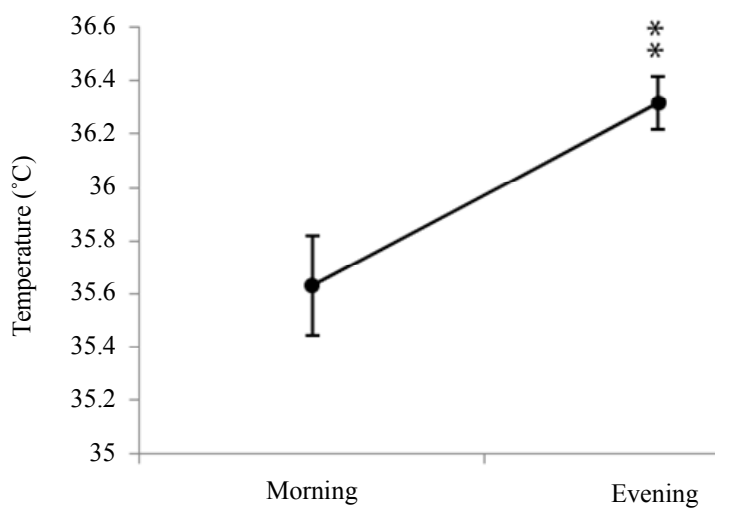

Figure 1.

Time of day effect in intra-aural temperature. ${ }^{* *} P<0.01$. Significantly different from morning.
Table 1.

Mean $( \pm \mathrm{SD})$ values for Zigzag test (s), kicking accuracy $(\mathrm{cm})$, Ball control with the body, Ball control with the head (number of hits) and coordination measured in the morning and in the afternoon.

\begin{tabular}{lcc}
\hline \multicolumn{1}{c}{ Test } & $\mathbf{0 7 : 0 0 ~ h r}$ & $\mathbf{1 7 : 0 0 ~ h r}$ \\
\hline Zigzag with the ball (s) & $7.69 \pm 0.32$ & $7.36 \pm 0.31^{*}$ \\
Zigzag test (s) & $5.92 \pm 0.32$ & $5.55 \pm 0.21^{* *}$ \\
kicking accuracy (cm) & $46.7 \pm 12.2$ & $43.1 \pm 13.1$ \\
$\begin{array}{l}\text { Ball control with the body } \\
\text { (number of hits) }\end{array}$ & $32.53 \pm 19.93$ & $36.07 \pm 30.11$ \\
$\begin{array}{l}\text { Ball control with the head } \\
\text { (number of hits) }\end{array}$ & $6.33 \pm 3.09$ & $7.27 \pm 3.39$ \\
$\begin{array}{l}\text { Skill index } \\
\text { Coordination }\end{array}$ & $0.77 \pm 0.02$ & $0.75 \pm 0.02$ \\
chest-foot-head (points) & $2.33 \pm 1.11$ & $2.07 \pm 1.22$ \\
head- foot- foot (points) & $1.53 \pm 0.83$ & $1.40 \pm 0.83$ \\
foot-chest-head (points) & $0.80 \pm 0.56$ & $0.73 \pm 0.70$ \\
\hline
\end{tabular}

Note: ${ }^{* * * *}$ significant difference in comparison with $07: 00 \mathrm{~h}$ at the levels of $P<$ 0.05 and $P<0.01$.

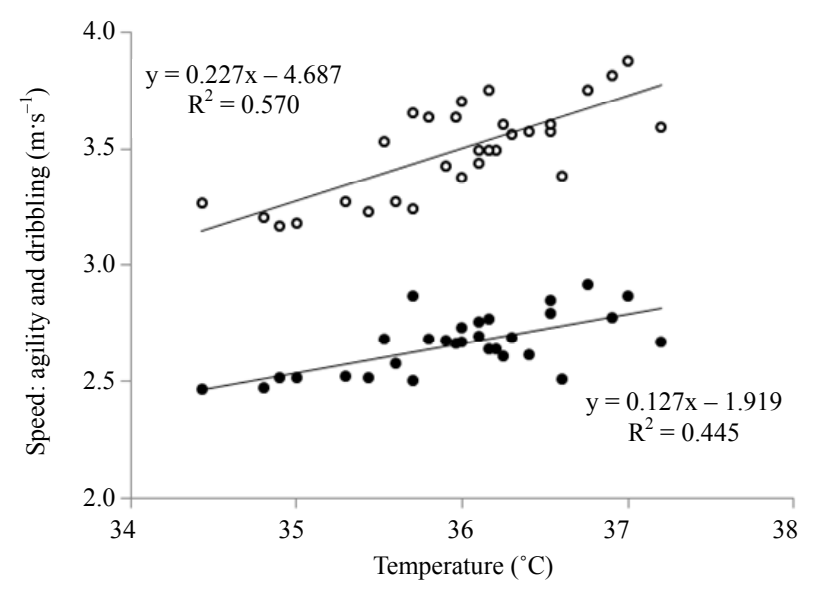

Figure 2.

Correlation between intra-aural temperature and speed for dribbling (closed circles) and agility (open circles).

the gains observed in our experimental population (around $0.68^{\circ} \mathrm{C}$ ) are in accordance with the amplitude (peak-to-trough variation) demonstrated on boys which showed an amplitude ranging from $0.5^{\circ} \mathrm{C}$ to $1^{\circ} \mathrm{C}$ (Melhim et al., 1993; Souissi et al., 2010; Zarrouk et al., 2012).

The results of the present investigation, focused on the diurnal fluctuations of boy's footballers, showed that agility and dribbling fluctuate with time-of-day, with morning nadirs, evening highest values and an amplitude equal to $8 \% \pm 6.7 \%$ and $4.5 \% \pm 5.9 \%$, respectively. The worst performance of dribbling and agility observed in the morning can be explained by the significant low body-temperature $(P<0.01)$ which can modify muscular electric activity, causing a change in muscular coordination and, consequently, a performance decrease (Oksa et al., 1996). Moreover, the effect of time of day on muscle contractile properties could be attributed, in part, to an intracellular 
variation in the muscle (e.g., a circadian variation in inorganic phosphate concentration) and/or, in part, to the circadian rhythm in the central temperature, which could influence calcium release by the sarcoplasmic reticulum (Guette et al., 2005).

The findings of this study were in agreement with previous studies who reported that physical (Chtourou et al., 2012a, 2012b; Hamouda et al., 2012; Aloui et al., 2012; Reilly et al., 2007, Bernard et al., 1998; Melhim et al., 1993) and some specific skill soccer (Reilly et al., 2004a, 2004b, 2007) performances were significantly higher in the evening than the morning (Chtourou et al., 2012a, 2012b; Hamouda et al., 2012; Aloui et al., 2012; Reilly et al., 2007, Bernard et al., 1998; Melhim et al., 1993). Nevertheless, it was reported that time of day have no effect on dribbling time (Reilly et al., 2004a, 2007). The disagreement with our study is probably due to differences in the study protocol, as the distance dribble $(40 \mathrm{~m})$ is higher than the present one $(20 \mathrm{~m})$.

A major finding of the present study was that the high intra aural temperature was associated with a significant increase in agility and dribble performance. It would seem, therefore, that changes in some skills soccer performance are justified by changes in body temperature during the course of the day time. Similar findings were reported in swimming (Zarrouk et al., 2012), running (Chtourou et al., 2012b), tennis (Drust et al., 2005), football (Reilly et al., 2004a, 2004b, 2007), badminton (Edwards et al., 2005). It can, thus, be concluded that there is a circadian rhythm in some specific skills performance in boy's footballer. Indeed, the association between the diurnal temperature variation and the different aspects of physical (Souissi et al., 2010; Winget et al., 1985), mental (Reilly et al., 2007), perceptual, sensory motor, psychomotor (Weipeng et al., 2011) and cognitive performances (Winget et al., 1985) were found on many studies in adults (Reilly et al., 2007; Winget et al., 1985) and in children ((Souissi et al., 2010) performances. Usually, peak performances were found to occur in the early evening and correspond to the maximum body (Aloui et al., 2012; Reilly et al., 2007, Bernard et al., 1998; Melhim et al., 1993; Souissi et al., 2010). Although the exact mechanisms explaining this relationship remain unclear, it has been suggested that the increase in body temperature allows an increase in energy metabolism, improved muscle compliance, facilitate actin-myosin crossbridging (Weipeng et al., 2011), enhance metabolic reactions, increase the extensibility of connective tissue, reduce muscle viscosity and increase conduction velocity of action potentials (Sedliak et al., 2007). Even though it is still largely accepted that temperature is the primary circadian indicator of physical performance, recent evidence has challenged the traditional views about the relationship between body temperature and exercise performance. Recent studies investigating the neuromuscular performance at different timeof-day revealed a distinct circadian rhythm in physiological variables independent of temperature changes (Guette et al., 2005). This can explain the fact that circadian rhythm in sporting performance is affected by multi-factorial mechanisms, such as time since waking, level of fatigue, alertness (Atkinson et al., 1998; Edwards et al., 2007) and mood state (Drust et al., 2005). Therefore, the elucidation of these mechanisms is extremely complicated to be attributed to one variable. Future studies should investigate the complexity of these variables.

Nevertheless, there was no significant time of day effect on juggling (i.e., Ball control with the body, ball control with the head), coordination tests, skill index and kicking accuracy (Table 1). This might reflect in some way the level of skill and the modest experience (4.2 years) of the players recruited for this study. In addition, This finding can be related to the fact, as mentioned in the methods sections, that the measurements of the specific tests used were taken only at two times of day: 07:00 and 17:00 $\mathrm{h}$ and, therefore, we could not determine the exact time of peak specific technical skills performance (i.e., coordination, control with body and with head and kicking accuracy). Measuring performance continuously is not easy to implement and these time points (07:00 and 17:00 h) were chosen as they are generally reported in the literature (Chtourou et al. 2012a, 2012b; Hamouda et al., 2012; Aloui et al., 2012). Our results differ from those of previous studies which showed that the accuracy and consistency of badminton (Edwards et al., 2005), tennis serves (Atkinson et al., 1998), shooting accuracy (Reilly et al., 2004b) and juggling (Reilly et al., 2007) seems to be time of day dependent with higher accuracy observed in the evening (Reilly et al., 2004b) and in the middle of the day (Drust et al., 2005; Edwards et al., 2005). Circadian rhythms are also observed for other soccer-specific performance tests such as chipping, dribbling, and juggling. The discrepancy between our results and those of many workers (Reilly et al., 2004b; Drust et al., 2005; Edwards et al., 2005), could be in part accounted for the differences in the task performed and muscle groups solicited (upper vs. lower extremities), the ways of the accuracy measurements (radial error, distance from the target, number of points, scores per trial vs. mean error), the type of projectile (arrow and tennis ball vs. soccer ball), the distance between target and service line or ball position $(2.37 \mathrm{~m}$, $3.56 \mathrm{~m}$. vs. $6.1 \mathrm{~m}$ ), the age (adults vs. boys), and the specificity of sport (racquet sport vs. football).

Although the precise mechanisms of this response require further investigation, coaches and sport scientists should consider these findings when attempting to develop the specific skills performance for soccer players. In other respects, from the results of the present study, it is understood that the ability of some skills at different times of the day varies, due to the circadian rhythmicity.

\section{Conclusion}

An increase of intra-aural temperature, agility and dribbling were shown in the evening compared to morning values. The measures showed the best performance at 17:00 $\mathrm{h}$ and the intra-aural temperature fluctuates concomitantly. No time-of-day effect was observed for the ball control with the body, ball control with the head, coordination tests, skill Index and kicking accuracy performance.

\section{REFERENCES}

Aloui, A., Chaouachi, A., Chtourou, H., Wong, D., Haddad, M., Chamari, K., \& Souissin, N. (2012). Effects of Ramadan on the diurnal variations of repeated sprints performances. International Journal of Sports Physiology and Performance (in press).

Atkinson, G., \& Spiers, L. (1998). Dirunal variations in tennis serve. Perceptual and Motor Skills, 86, 1335-1338. doi: $10.2466 /$ pms.1998.86.3c.1335

Bernard, T., Giacomoni, M., Gavarry, O., Seymat, M., \& Falgairette, G. (1998). Time-of-day effects in maximal anaerobic leg exercise. European Journal of Applied Physiology and Occupational Physiology, 77, 133-138. doi:10.1007/s004210050311 
Chtourou, H., Hammouda, O., Chaouachi, A., Chamari, K., \& Souissi, N. (2012a). The effect of time-of-day and Ramadan fasting on anaerobic performances. International Journal of Sports Medicine, 33, 142-147. doi:10.1055/s-0031-1286251

Chtourou, H., Hammouda, O., Souissi, H., Chamari, K., Chaouachi, A., \& Souissi, N. (2012b). Diurnal variations in physical performances related to football in adolescent soccer players. Asian Journal of Sports Medicine, 3, 139-144.

Drust, B., Waterhouse, J., Atkinson, G., Edwards, B., \& Reilly, T. (2005). Circadian rhythms in sports performance-an update. Chronobiology International, 22, 21-44. doi:10.1081/CBI-200041039

Edwards, B., Lindsay, L., \& Waterhouse, J. (2005). Effect of time of day on the accuracy and consistency of the badminton serve. Ergonomics, 48, 1488-1498. doi:10.1080/00140130500100975

Edwards, B., Waterhouse, J., \& Reilly, T. (2007). The effects of circadian rhyhmicity and time-awake on a simple motor task. Chronobiology International, 24, 1109-1124. doi: $10.1080 / 07420520701795316$

Finnoff, J. T., Newcomer, K., \& Laskowski, E. R. (2002). A valid and reliable method for measuring the kicking accuracy of soccer players. Journal of Science and Medicine in Sport, 5, 348-353. doi:10.1016/S1440-2440(02)80023-8

Guette, M., Gondin, J., \& Martin, A. (2005). Time-of-day effect on the torque and neuromuscular properties of dominant and nondominant quadriceps femoris. Chronobiology International, 22, 541-558. doi:10.1081/CBI-200062407

Hamouda, O., Chtourou, H., Farjallah, M. A., Damien, D., \& Souissi, N. (2012). The effect of Ramadan fasting on the diurnal variations in aerobic and anaerobic performances in Tunisian youth soccer players. Biological Rhythm Research, 43, 177-190. doi:10.1080/09291016.2011.560050

Horne, J. A., \& Ostberg, O. (1976). A self-assessment questionnaire to determine morningness-eveningness in human circadian rhythms. International Journal of Chronobiology, 4, 97-110. doi:10.3109/07420529709001458

Huguet G., Touitou Y., Reinberg A. (1995). Diurnal Changes in Sport Performance of 9 - 11-Year-Old School Children. Chronobiology International, 14, 371-384.

Little, T., \& Williams, A. G. (2005). Specificity of acceleration, maximum speed, and agility in professional soccer players. Journal of Strength and Conditioning, 19, 76-78.

Malina, R. M., Cumming, S. P., Kontos, A. P., Eisenmenn, J. C., Ribeiro, B., \& Aroso, J. (2005). Maturity-associated variation in sport-specific skills of youth soccer players aged 13 - 15 years. Journal of Sports Sciences, 23, 515-522.

doi:10.1080/02640410410001729928

Melhim, A. F. (1993). Investigation of circadian rhythms in peak power and mean power of female physical education students. International Journal of Sports Medicine, 14, 303-306. doi:10.1055/s-2007-1021182

Mirkov, D. M., Nedeljkovic, A., Kukolj, M., Ugarkovic, D., \& Jaric, S. (2008). Evaluation of reliability of soccer-specific field tests. Journal of Strength and Conditioning, 22, 1046-1050. doi:10.1519/JSC.0b013e31816eb4af

Oksa, J., Rintamaki, H., Makinen, T., Martikkala, V., \& Rusko, H. (1996). EMG activity and muscular performance of lower leg during stretch-shortening cycle after cooling. Acta Physiologica Scandinavica, 157, 71-78. doi:10.1046/j.1365-201X.1996.452172000.x

Reilly, T., Atkinson, G., Edwards, B., Waterhouse, J., Farrelly, K., \& Fairhurst, E. (2007). Diurnal variation in temperature, mental and physical performance, and tasks specifically related to football (soccer). Chronobiology International, 224, 507-519. doi: 10.1080/07420520701420709

Reilly, T., Fairhurst, E., Edwards, B., \& Waterhouse, J. (2004a). Time of day and performance tests in male football players. In T. Reilly, J. Cabri, \& D. Araujo (Eds.), Science and Football V. London Routledge, 275-279.

Reilly, T., Farrelly, K., Edwards, B., \& Waterhouse, J. (2004b). Effects of time of day on the performance of soccer-specific motor skills. In T. Reilly, J. Cabri, \& D. Araujo (Eds.), Science and Football V. London: Routledge, 272-274.

Rosch, D., Hodgson, R., Peterson, T. L., Graf-Baumann, T., Junge, A., Chomiak, J., \& Dvorak, J. (2000). Assessment and evaluation of football performance. American Journal of Sports Medicine, 28, 29-39.

Sedliak, M., Finni, T., Cheng, S., Kraemer, W. J., \& Häkkinen, K. (2007). Effect of time-of-day-specific strength training on serum hormone concentrations and isometric strength in men. Chronobiology International, 224, 1159-1177. doi: $10.1080 / 07420520701800686$

Souissi, H., Chaouachi, A., Chamari, K., Dogui, M., Amri, M., \& Souissi, N. (2010). Time-of-day effects on short-term exercise performances in 10 - 11-years-old Boys. Pediatric Exercise Science, 22, 613-623.

Weipeng, T., Michael, J., \& Michael, R. (2011). Circadian rhythms in exercise performance: Implications for hormonal and muscular adaptation. Journal of Sports Science and Medicine, 10, 600-606.

Winget, C. M., DeRoshia, C. W., \& Holley, C. (1985). Circadian rhythms and athletic performance. Medicine and Science in Sports and Exercise, 17, 498-516. doi:10.1249/00005768-198510000-00002

Zarrouk, N., Chtourou, H., Rebai, H., Hammouda, O., Souissi, N., Dogui, M., \& Hug, F. (2012). Time of day effects on repeated sprint ability. International Journal of Sports Medicine, 33, 975-980. doi: $10.1055 / \mathrm{s}-0032-1312626$ 